\title{
T6SS Accessory Proteins, Including DUF2169 Domain-Containing Protein and Pentapeptide Repeats Protein, Contribute to Bacterial Virulence in T6SS Group_5 of Burkholderia glumae BGR1
}

\author{
Namgyu Kim ${ }^{1}$, Gil Han ${ }^{1}$, Hyejung Jung ${ }^{1}$, Hyun-Hee Lee ${ }^{1}$, Jungwook Park ${ }^{2}$ and Young-Su Seo ${ }^{1, *}$ \\ 1 Department of Integrated Biological Science, Pusan National University, Busan 46241, Korea; \\ titanic622@pusan.ac.kr (N.K.); croone@pusan.ac.kr (G.H.); jhj4059@pusan.ac.kr (H.J.); \\ ehyuna92@pusan.ac.kr (H.-H.L.) \\ 2 Environmental Microbiology Research Team, Nakdonggang National Institute of Biological, \\ Resources (NNIBR), Sangju 37242, Korea; jjuwoogi@nnibr.re.kr \\ * Correspondence: yseo2011@pusan.ac.kr
}

check for updates

Citation: Kim, N.; Han, G.; Jung, H.; Lee, H.-H.; Park, J.; Seo, Y.-S. T6SS Accessory Proteins, Including DUF2169 Domain-Containing Protein and Pentapeptide Repeats Protein, Contribute to Bacterial Virulence in T6SS Group_5 of Burkholderia glumae BGR1. Plants 2022, 11, 34. https:// doi.org/10.3390/plants11010034

Academic Editor: Tika Adhikari

Received: 23 November 2021

Accepted: 21 December 2021

Published: 23 December 2021

Publisher's Note: MDPI stays neutral with regard to jurisdictional claims in published maps and institutional affiliations.

Copyright: (C) 2021 by the authors. Licensee MDPI, Basel, Switzerland. This article is an open access article distributed under the terms and conditions of the Creative Commons Attribution (CC BY) license (https:// creativecommons.org/licenses/by/ $4.0 /)$.

\begin{abstract}
Burkholderia glumae are bacteria pathogenic to rice plants that cause a disease called bacterial panicle blight (BPB) in rice panicles. $\mathrm{BPB}$, induced by B. glumae, causes enormous economic losses to the rice agricultural industry. B. glumae also causes bacterial disease in other crops because it has various virulence factors, such as toxins, proteases, lipases, extracellular polysaccharides, bacterial motility, and bacterial secretion systems. In particular, B. glumae BGR1 harbors type VI secretion system (T6SS) with functionally distinct roles: the prokaryotic targeting system and the eukaryotic targeting system. The functional activity of T6SS requires 13 core components and T6SS accessory proteins, such as adapters containing DUF2169, DUF4123, and DUF1795 domains. There are two genes, bglu_1g23320 and bglu_2g07420, encoding the DUF2169 domain-containing protein in the genome of B. glumae BGR1. bglu_2g07420 belongs to the gene cluster of T6SS group_5 in B.glumae BGR1, whereas bglu_1g23320 does not belong to any T6SS gene cluster in B. glumae BGR1. T6SS group_5 of B. glumae BGR1 is involved in bacterial virulence in rice plants. The DUF2169 domaincontaining protein with a single domain can function by itself; however, $\Delta u 1 g 23320$ showed no attenuated virulence in rice plants. In contrast, $\Delta u 2 g 07420 D U F 2169$ and $\Delta u 2 g 07420 P P R$ did exhibit attenuated virulence in rice plants. These results suggest that the pentapeptide repeats region of the C-terminal additional domain, as well as the DUF2169 domain, is required for complete functioning of the DUF2169 domain-containing protein encoded by bglu_2g07420. bglu_2g07410, which encodes the pentapeptide repeats protein, composed of only the pentapeptide repeats region, is located downstream of bglu_2g07420. $\Delta u 2 g 07410$ also shows attenuated virulence in rice plants. This finding suggests that the pentapeptide repeats protein, encoded by bglu_2g07410, is involved in bacterial virulence. This study is the first report that the DUF2169 domain-containing protein and pentapeptide repeats protein are involved in bacterial virulence to the rice plants as T6SS accessory proteins, encoded in the gene cluster of the T6SS group_5.
\end{abstract}

Keywords: type VI secretion system; T6SS accessory proteins; T6SS adaptor; DUF2169 domain; pentapeptide repeats-containing protein

\section{Introduction}

Burkholderia glumae was first reported in Japan in the 1950s [1]. B. glumae are bacteria pathogenic to rice plants, which cause a disease called bacterial panicle blight (BPB) in rice panicles [2,3]. B. glumae has several virulence factors, such as toxins, proteases, lipases, extracellular polysaccharides, bacterial motility, and bacterial secretion systems [4-10]. Thus, B. glumae not only cause BPB in rice panicles, but also bacterial wilt in rice stems and various other economically important crops, such as tomatoes, eggplants, hot peppers, and 
sesame [3,4]. The virulence factors of B. glumae contribute to its resistance against the host immune system and cause damage the host. In particular, bacterial secretion systems have specialized, sophisticated, and highly developed strategies for the selective transport of toxic proteins (as effector proteins) across bacterial cell membranes. B. glumae BGR1 has four functionally distinct type VI secretion systems (T6SS): T6SS group_1, T6SS group_2, group_4, and T6SS group_5. T6SS group_1 of B. glumae BGR1 is involved in bacterial competition, whereas T6SS group_4 and group_5 contribute to bacterial virulence [3]. However, the type VI secreted proteins of B. glumae BGR1 have not clearly been identified.

One of the bacterial secretion systems, the type VI secretion system (T6SS), is a contractile nanomachine which is prevalent in $25 \%$ of Gram-negative bacteria and contributes to interspecies interaction in a contact-dependent manner with adjacent bacteria or eukaryotic cells [11-13]. T6SS is composed of 13 major components (TssA-TssM), assorted into specific structures, i.e., a cell-envelope-associated membrane complex, a baseplate complex, and a needle complex [14-16]. In addition to these major components, several accessory proteins related to T6SS, called T6SS accessory proteins, work with T6SS-dependent effectors to perform their functions.

A cell-envelope-associated membrane complex is assembled using TssM, TssL, and TssJ. The baseplate complex is assembled from TssE, TssF, and TssG, and the baseplate complex and membrane complex are connected by TssK. TssA is involved in baseplate assembly and TssB/TssC in sheath polymerization. The needle complex comprises TssD as an inner tube, a TssB/TssC sheath surrounding the inner tube, and a VgrG/PAAR spike complex at its end $[17,18]$. The VgrG/PAAR protein spike complex is formed of the VgrG trimer with PAAR and is responsible for creating an opening in the target cells [19-21]. The VgrG/PAAR protein spike complex acts on its own as an effector if it contains an additional C-terminal toxin domain [20]. Another effector, called the cargo effector, is bound through non-covalent interactions with structural components, such as VgrG, PAAR protein, and TssD, either directly, or with the aid of an adapter protein or chaperone [22-25]. A cargo effector is considered to be one of several T6SS-associated proteins. In particular, effectors that are supported by adaptor/chaperones, which are not secreted by the T6SS, are indispensable for mounting on the spike complex and for enabling secretion from bacterial cells [26].

T6SS-dependent effectors are injected directly into adjacent target cells or with the aid of T6SS accessory proteins, such as T6SS adaptor/chaperones [25,26]. T6SS adaptors/chaperones include specific protein domains, such as DUF1795, DUF4123, and DUF2169 domains. In addition, T6SS adaptor/chaperones interact with T6SS-dependent effectors and VgrG (TssI) based on protein-protein interactions. The chaperone/adaptor with the DUF4123 domain was identified through co-immunoprecipitation and bacterial two-hybrid analysis in Vibrio cholerae through its interactions with VgrG-1 and T6SS-dependent effectors (TseL) [27]. DUF4123 domain-containing proteins of $V$. cholerae are required for the secretion of TseL and TseL-mediated antibacterial activity [28]. However, the identity and function of T6SSdependent effectors are still not well understood.

In this study, DUF2169 domain-containing protein and pentapeptide repeats protein, which is involved in the bacterial virulence activity of B. glumae BGR1, was identified. The genes of $b g l u \_2 g 07420$ and $b g l u \_2 g 07410$, the functionally unidentified genes encoding these two proteins, are present in the gene cluster of T6SS group_5 in B glumae BGR1. Among them, bglu_2g07420 encodes the region of the DUF2169 domain, known as T6SS adapter/chaperones at its N-terminus and the region of pentapeptide repeats at its Cterminus. In addition, bglu_1g23320, which encodes only the DUF2169 domain in the genome of B. glumae BGR1, does not belong to the four T6SS gene clusters in B. glumae BGR1. Only loss of the DUF2169 domain of bglu_2g07420 belonging to the gene cluster of T6SS group_5 showed attenuated virulence in rice plants. In addition, the bacterial virulence of $B$. glumae BGR1 was found to be affected by the loss of the pentapeptide repeats region in $b g l u \_2 g 07420$ and $b g l u \_2 g 07410$. bglu_2g07410 encodes the pentapeptide repeats protein with only the pentapeptide repeats region. Our findings show that bglu_2g07420 
and bglu_2g07410, belonging to the gene cluster of T6SS group_5 in B. glumae BGR1, encode the DUF2169 domain-containing protein and pentapeptide repeats protein, respectively, and contribute to the bacterial virulence to rice plants as the T6SS-associated proteins.

\section{Results}

2.1. Investigation and Analysis of the Genes Containing the DUF2169 Domain and Its Adjacent Gene in the Genome of Burkholderia glumae BGR1

Some T6SS accessory proteins, such as DUF1795, DUF4123, and DUF2169 domaincontaining proteins, are known T6SS adapters that require the loading of a specific effector onto the cognate VgrG for delivery [25]. The DUF2169 domain-containing protein was found to be a T6SS accessory protein in plant pathogenic bacteria, A. tumefaciens; therefore, we focused on only the genes encoding the DUF2169 domain of the T6SS accessory protein [22]. The only two genes encoding the DUF2169 domain in the genome of B. glumae BGR1, bglu_1g23320 and bglu_2g07420, were identified using the National Center for Biotechnology Information (https:/ / www.ncbi.nlm.nih.gov/protein; accessed on 17 August 2021). In particular, bglu_2g07420 was present in the gene cluster of T6SS group_5 in B. glumae BGR1 (Figure 1A). However, bglu_1g23320 was not included in the gene cluster of any T6SS group and existed independently as a separate entity (Figure 1B). The bglu_1g23320 gene is a hypothetical protein (WP_015876299.1) composed of 362 amino acids, whereas bglu_2g07420 is a hypothetical protein (WP_012733555.1) composed of 878 amino acids. Functional analysis of the proteins encoded by the two genes was performed using the InterPro database (http: / / www.ebi.ac.uk/interpro; accessed on 17 August 2021) (Table 1). The $b g l u \_1 g 23320$ gene encodes only the DUF2169 domain, whereas the $b g l u \_2 g 07420$ gene encodes the DUF2169 domain at the N-terminus and the pentapeptide repeats region at the C-terminus (Figure 2). Additionally, the non-annotated gene, bglu_2g07410, located adjacent to bglu_2g07420, encoding the DUF2169 domain-containing protein, was also analyzed with the InterPro database. The $b g l u \_2 g 07410$ gene, which is located downstream of the $b g l u \_2 g 07420$ gene, was evaluated as a pentapeptide repeats protein (WP_012733554.1), encoding only the pentapeptide repeats region consisting of 356 amino acids (Figure 1A and Table 1). We assumed that the bglu_2g07420 gene, which is present in the gene cluster of T6SS group_5, and the other gene of bglu_1g23320, which is not present in any T6SS group, are the major putative T6SS accessory proteins involved in the function of T6SS group_5 in B. glumae BGR1. Furthermore, we assumed that the bglu_2g07410 gene encoding the pentapeptide repeats region, similarly to the $b g l u \_2 g 07420$ gene with its pentapeptide repeats region, is involved in the function of T6SS group_5 as a putative T6SS accessory protein.

A

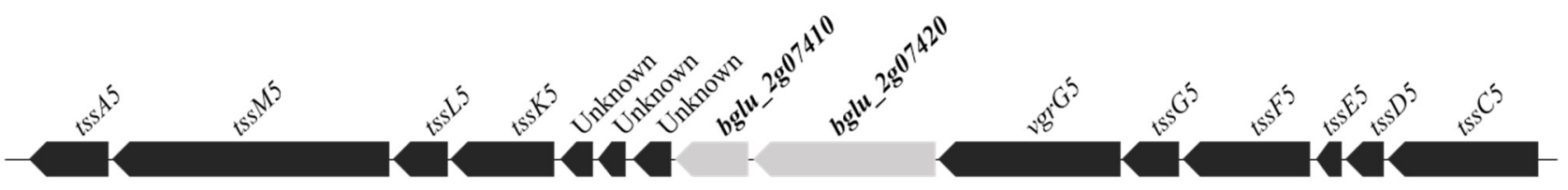

B

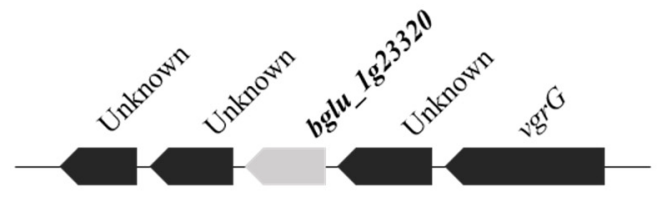

Figure 1. Genetic organization of T6SS group_5, containing bglu_2g07420 encoding the DUF2169containing protein and $b g l u \_2 g 07410$ encoding pentapeptide repeats protein (A), and $b g l u \_1 g 23320$ encoding the DUF2169-containing protein (B) in Burkholderia glumae BGR1. The genes are indicated by the locus ID (e.g., bglu_2g07410) and are to scale. The genes marked in gray encode the DUF2169 domain-containing proteins and pentapeptide repeats protein. 
Table 1. Functional analysis of WP_015876299.1,WP_012733554.1, and WP_012733555.1 from the InterPro database.

\begin{tabular}{|c|c|c|c|c|c|c|}
\hline Analysis & Accession & Description & $\begin{array}{l}\text { InterPro } \\
\text { Accession }\end{array}$ & $\begin{array}{c}\text { InterPro } \\
\text { Description }\end{array}$ & Interval & E-Value \\
\hline \multicolumn{7}{|c|}{ WP_015876299.1 } \\
\hline Pfam & PF09937 & $\begin{array}{c}\text { Uncharacterized } \\
\text { protein conserved in } \\
\text { bacteria (DUF2169) }\end{array}$ & IPR018683 & DUF2169 & $21 \sim 311$ & $5.6 \times 10^{-91}$ \\
\hline \multicolumn{7}{|c|}{ WP_012733554.1 } \\
\hline Pfam & PF13599 & $\begin{array}{l}\text { Pentapeptide repeats } \\
\text { (9 copies) }\end{array}$ & IPR001646 & Pentapeptide repeat & $66 \sim 137$ & $2.5 \times 10^{-10}$ \\
\hline Pfam & PF00805 & $\begin{array}{l}\text { Pentapeptide repeats } \\
\text { (8 copies })\end{array}$ & IPR001646 & Pentapeptide repeat & 193 230 & $3.2 \times 10^{-9}$ \\
\hline Pfam & PF13599 & $\begin{array}{c}\text { Pentapeptide repeats } \\
\text { (9 copies) }\end{array}$ & IPR001646 & Pentapeptide repeat & $238 \sim 308$ & $1.7 \times 10^{-7}$ \\
\hline \multicolumn{7}{|c|}{ WP_012733555.1 } \\
\hline Pfam & PF09937 & $\begin{array}{l}\text { Uncharacterized } \\
\text { protein conserved in } \\
\text { bacteria (DUF2169) }\end{array}$ & IPR018683 & DUF2169 & 20 307 & $6.4 \times 10^{-73}$ \\
\hline Pfam & PF00808 & $\begin{array}{l}\text { Pentapeptide repeats } \\
\text { (8 copies })\end{array}$ & IPR001646 & Pentapeptide repeat & $605 \sim 642$ & $2.0 \times 10^{-11}$ \\
\hline Pfam & PF13599 & $\begin{array}{l}\text { Pentapeptide repeats } \\
\text { (9 copies })\end{array}$ & IPR001646 & Pentapeptide repeat & $801 \sim 864$ & $3.1 \times 10^{-6}$ \\
\hline
\end{tabular}

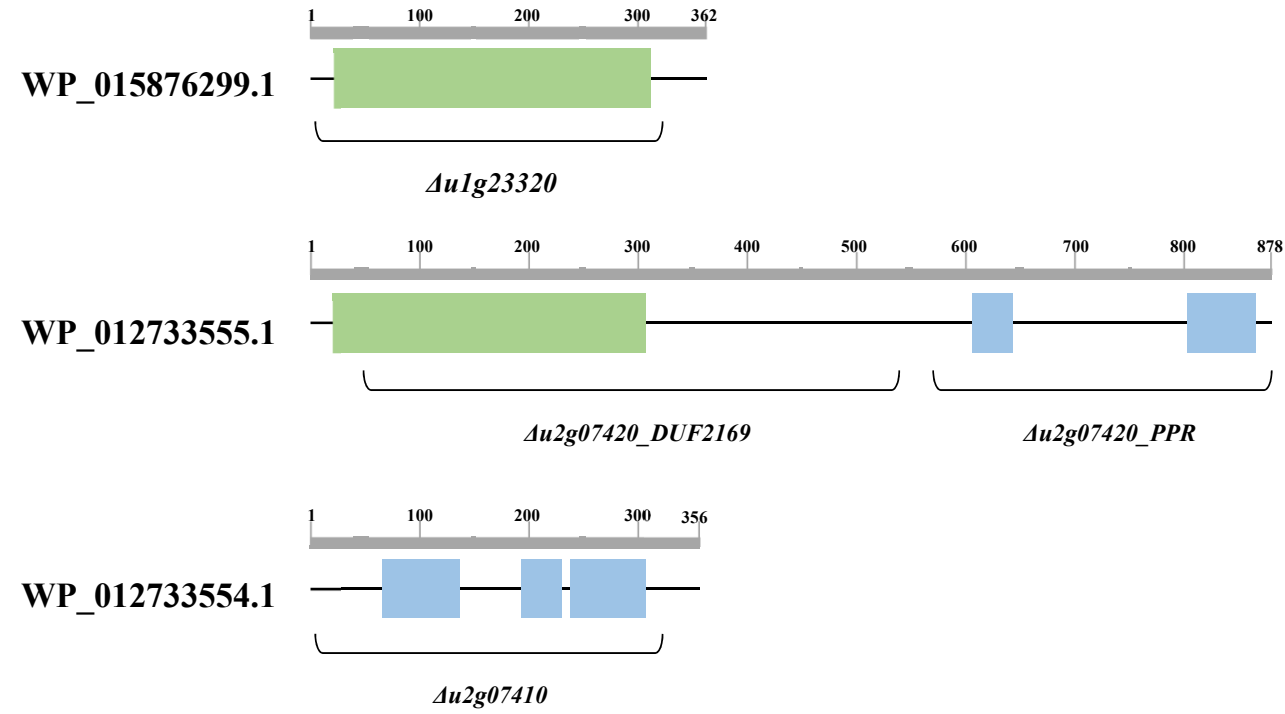

\section{DUF2169 domain Pentapeptide repeats region}

Figure 2. Domain architectures of WP_015876299.1,WP_012733554.1, and WP_012733555.1. Only proteins with distinct domain organizations are shown. The DUF2169 domain-containing protein (WP_015876299.1) encoded by bglu_1g23320 has the DUF2169 domain as a single-domain protein. The DUF2169 domain-containing protein (WP_012733555.1) encoded by bglu_2g07420 has multiple domains with pentapeptide repeats region at the C-terminus. The pentapeptide repeats protein (WP_012733554.1) encoded by bglu_2g07410 only has the pentapeptide repeats region. The deletion sites of the mutant strains used in this study are shown with $\Delta u 1 g 23320, \Delta u 2 g 07420 D U F 2169$, $\triangle u 2 g 07420 P P R$, and $\triangle u 2 g 07410$. 
2.2. Construction of Markerless Deletion Mutants to Evaluate Whether the Putative T6SS Accessory Protein Affects the Function of T6SS Group_5 in B. glumae BGR1

To investigate the functions of putative T6SS accessory proteins containing the DUF2169 domain, we constructed markerless deletion mutants targeting the DUF2169 domain of bglu_1g23320 and bglu_2g07420 genes via homologous recombination. Single markerless deletion mutants, called $\Delta u 2 g 07420 D U F 2169$ and $\Delta u 1 g 23320$, were generated via the deletion of only the DUF2169 domain of bglu_2g07420 and bglu_1g23320, respectively (Supplementary Tables S1 and S2). To complement the phenotypes of $\Delta u 2 g 07420 D U F 2169$ and $\Delta u 1 g 23320$, the respective complementation strains, $\Delta u 2 g 07420 D U F 2169-C$ and $\Delta u 1 g 23320$ $\mathrm{C}$, were generated.

To investigate whether the pentapeptide repeats region encoded in bglu_2g07410 and C-terminus of bglu_2g07420 affect the function of T6SS group-5 as putative T6SS accessory proteins, the pentapeptide repeats region of both genes was deleted to construct markerless deletion mutants. Single markerless deletion mutants, called $\Delta u 2 g 07420 P P R$ and $\Delta u 2 g 07410$, were generated by deleting the pentapeptide repeats region of bglu_2g07420 and bglu_2g07410, respectively (Supplementary Tables S2 and S3). To complement the phenotypes of $\triangle u 2 g 07420 P P R$ and $\Delta u 2 g 07410$, the respective complementation strains, $\triangle u 2 g 07420 P P R-C$ and $\triangle u 2 g 07420-C$, were generated. A double markerless deletion mutant, $\Delta u 2 g 07410-20$, was generated to determine whether two separate pentapeptide repeats regions independently contributed to the function of T6SS group_5. To complement the phenotype of $\Delta u 2 g 07410-20$, the respective complementation strain, $\Delta u 2 g 07410-20-\mathrm{C}$, was generated.

2.3. The DUF2169 Domain Encoded by the N-terminus of bglu_2g07420 inside the Gene Cluster of T6SS Group_5 Is Involved in Bacterial Virulence in Rice Plants

To determine whether the DUF2169 domain encoded by bglu_1g23320 and bglu_2g07420 is involved in the functional role of T6SS group_5 in bacterial virulence, causing bacterial panicle blight, rice plants at the flowering stage were infected with wild-type B. glumae BGR1 and the mutants $\Delta u 1 g 23320$ and $\Delta u 2 g 07420 D U F 2169$ (Figure 3). At 8 days post-inoculation (dpi), most of the wild-type BGR1-inoculated panicles had developed blight disease symptoms (severity, 0-5), with a disease severity score of $4.38 \pm 0.079$ for wild-type BGR1 (Figure 3B). In contrast, $\Delta u 2 g 07420 D U F 2169$ exhibited attenuated virulence, with a disease severity score of $2.73 \pm 0.134$ (Figure 3B). The restored strain, $2 g 07420 D U F 169-C$, completely recovered its virulence, with a disease severity score of $4.27 \pm 0.26$ (Figure 3B). Rice plants were also infected with the $\Delta u 1 g 23320$ mutant to determine whether the $b g l u \_1 g 23320$ gene contributed to bacterial virulence. However, the disease severity score of $\Delta u 1 g 23320$ was $4.26 \pm 0.046$ (Figure 3B). The deletion of the DUF2169 domain in bglu_1g23320 caused almost no difference in pathogenicity from wild-type BGR1, although the deletion of the DUF2169 domain in bglu_2g07420 differed from that of wild-type BGR1 (Figure 3). Thus, only the DUF2169 domain (WP_012733555.1), which is encoded by bglu_2g07420 in the gene cluster of T6SS group_5, is involved in bacterial virulence to rice plants. 
A

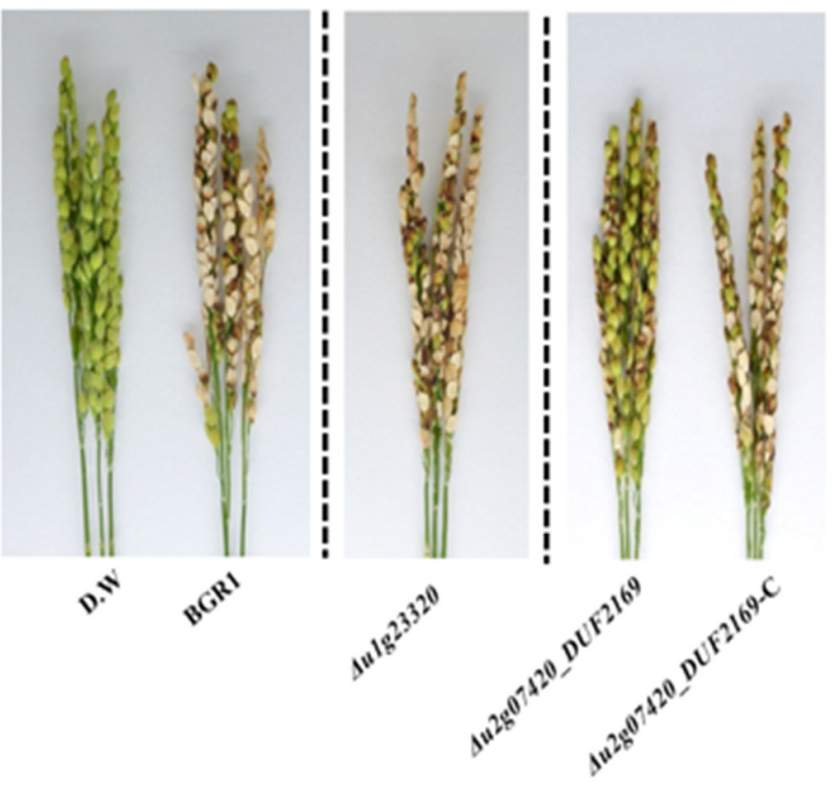

B

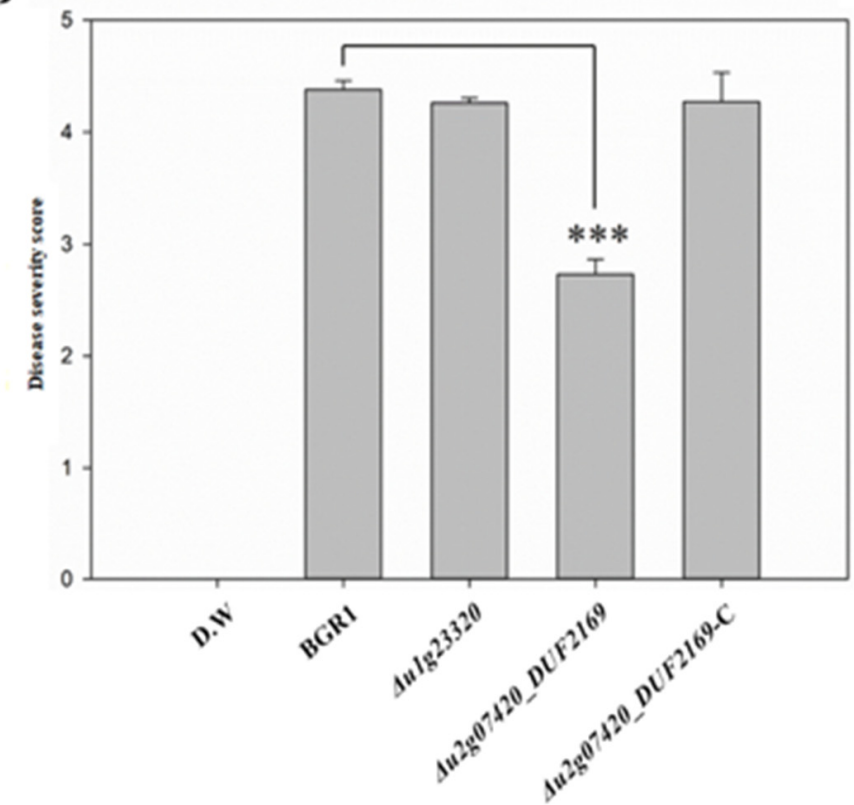

Figure 3. In vivo pathogenicity assay at the reproductive stage to assess the virulence of $\Delta u 1 g 23320$ and $\Delta u 2 g 07420 D U F 2169$. Representative of three replicates (A). Disease severity on the rice panicles was calculated on a scale of 0 to 5 after inoculating the bacterial suspension (B). Data are presented as the mean \pm S.D. of three replicates $(n=3)$. Mean values followed by the same letters are not significantly different according to Tukey's HSD test $\left.{ }^{* * *} p<0.001\right)$. Disease symptoms at 8 days post-inoculation. Distilled water (D.W.) was used as the negative control.

2.4. The Pentapeptide Repeats Region Encoded by bglu_2g07410 and the C-terminus of bglu_2g07420 inside the Gene Cluster of T6SS Group_5 Is Involved in Bacterial Virulence to Rice Plants

To determine whether only the DUF2169 domain encoded by the N-terminus of bglu_2g07420 is involved in the functional role of T6SS group_5, rice plants at the flowering stage were infected with $\Delta u 2 g 07420 P P R$, in which the C-terminal pentapeptide repeats region is deleted. At $8 \mathrm{dpi}$, most of the wild-type BGR1-inoculated panicles had developed blight disease symptoms (severity, 0-5), with a disease severity score of $4.38 \pm 0.079$ for wild-type BGR1 (Figure $4 \mathrm{~B}$ ). In contrast, $\triangle u 2 g 07420 P P R$ displayed attenuated virulence, with a disease severity score of $3.05 \pm 0.022$ (Figure 4B). To determine whether the pentapeptide repeats region is also involved in bacterial virulence, rice plants were infected with $\Delta u 2 g 07410$, in which $b g l u \_2 g 07410$ was deleted downstream of $b g l u \_2 g 07420$. $\Delta u 2 g 07410$ also showed attenuated virulence, with a disease severity score of $2.59 \pm 0.114$ (Figure 4B). $\Delta u 2 g 07410-20$ exhibited attenuated virulence, with a disease severity score of $1.85 \pm 0.091$ (Figure 4B), as did $\Delta u 2 g 07410-20$ (Figure 4B). The restored strains, namely, $\triangle u 2 g 07420 P P R-C, \Delta u 2 g 07410-C$, and $\Delta u 2 g 07410-20-C$, completely recovered their virulence, with disease severity scores of $4.32 \pm 0.279,4.26 \pm 0.193$, and $4.18 \pm 0.179$, respectively (Figure 4). The levels of attenuated virulence by single mutants, $\Delta u 2 g 07420$ and $\Delta u 2 g 07410$, were similar at the reproductive and flowering stages of rice plants (Figures 4 and 5). Thus, the pentapeptide repeats regions of $b g l u \_2 g 07420$ and $b g l u \_2 g 07410$ also contribute to bacterial virulence in rice plants. However, the results of in vivo pathogenicity assays at the reproductive stage showed that the bacterial virulence of $\Delta u 2 g 07410-20$, in which both genes were deleted, was more attenuated than that of single mutants (Figure 4 ). The mean disease severity score of $\Delta u 2 g 07410-20$ and the mean disease severity scores of $\Delta u 2 g 07410$ or $\Delta u 2 g 07420$ were compared using the least significant difference at $p<0.001$ according to Tukey's HSD test. 
A

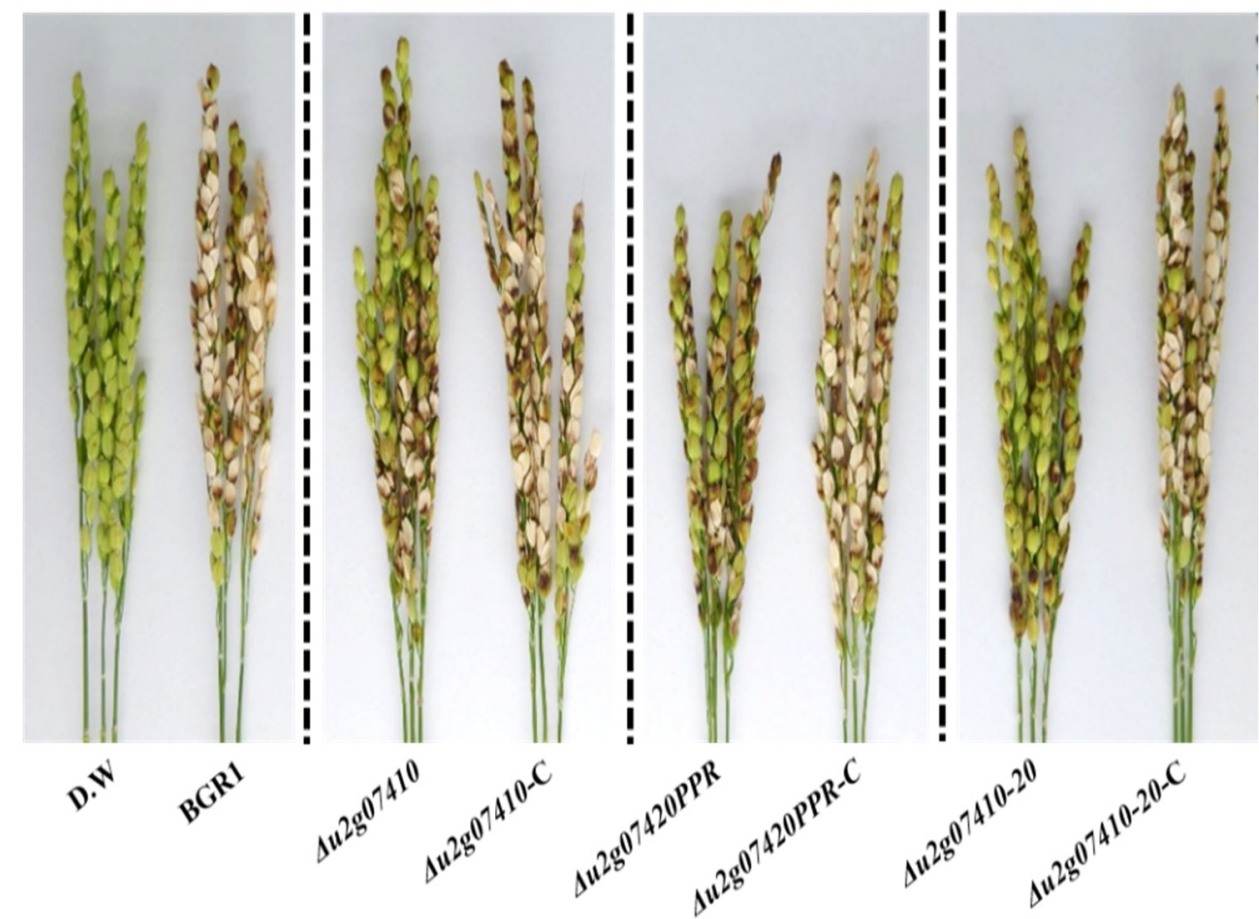

B

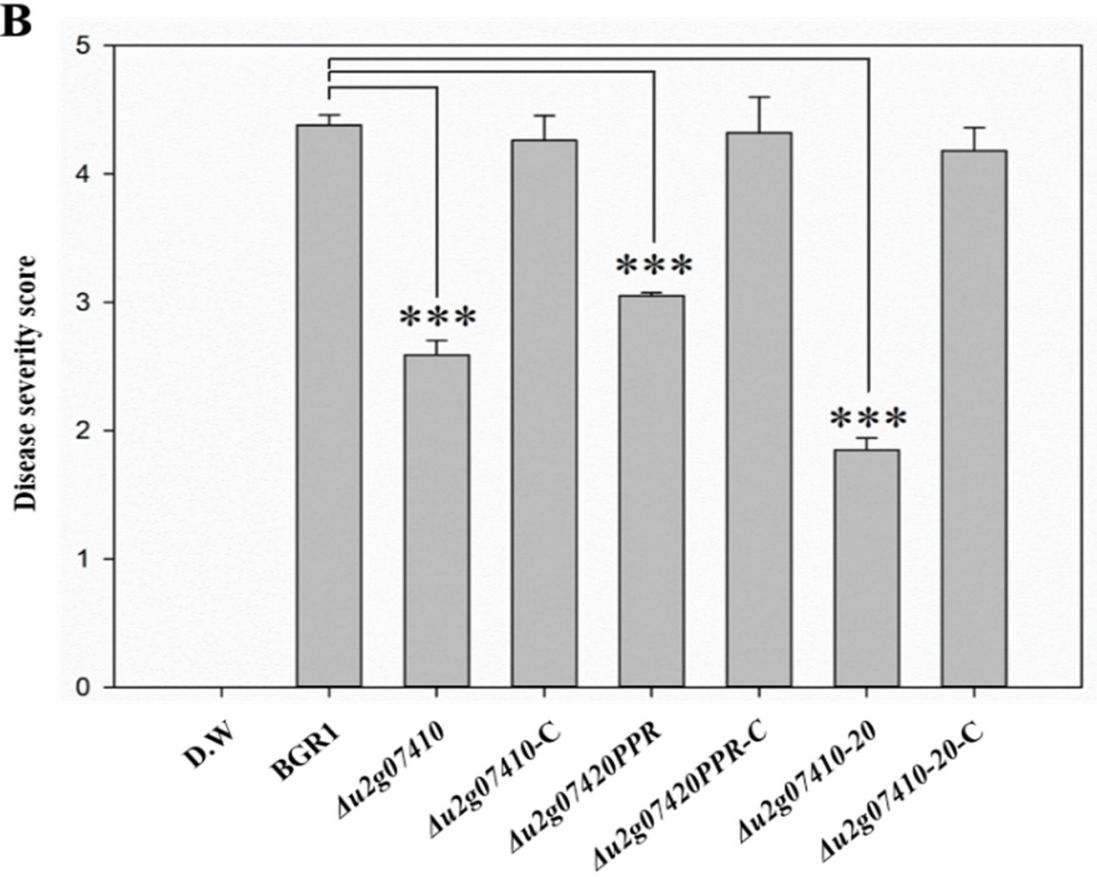

Figure 4. In vivo pathogenicity assay at the reproductive stage to assess the virulence of $\Delta u 2 g 07410$, $\triangle u 2 g 07420 P P R$, and $\Delta u 2 g 07410-20$. Representative of three replicates (A). Disease severity on the rice panicles was calculated on a scale of 0 to 5 after inoculating the bacterial suspension (B). Data are presented as the mean \pm S.D. of three replicates $(n=3)$. Mean values followed by the same letters are not significantly different according to Tukey's HSD test $(* * *<0.001)$. Disease symptoms at 8 days post-inoculation. Distilled water (D.W) was used as the negative control. 

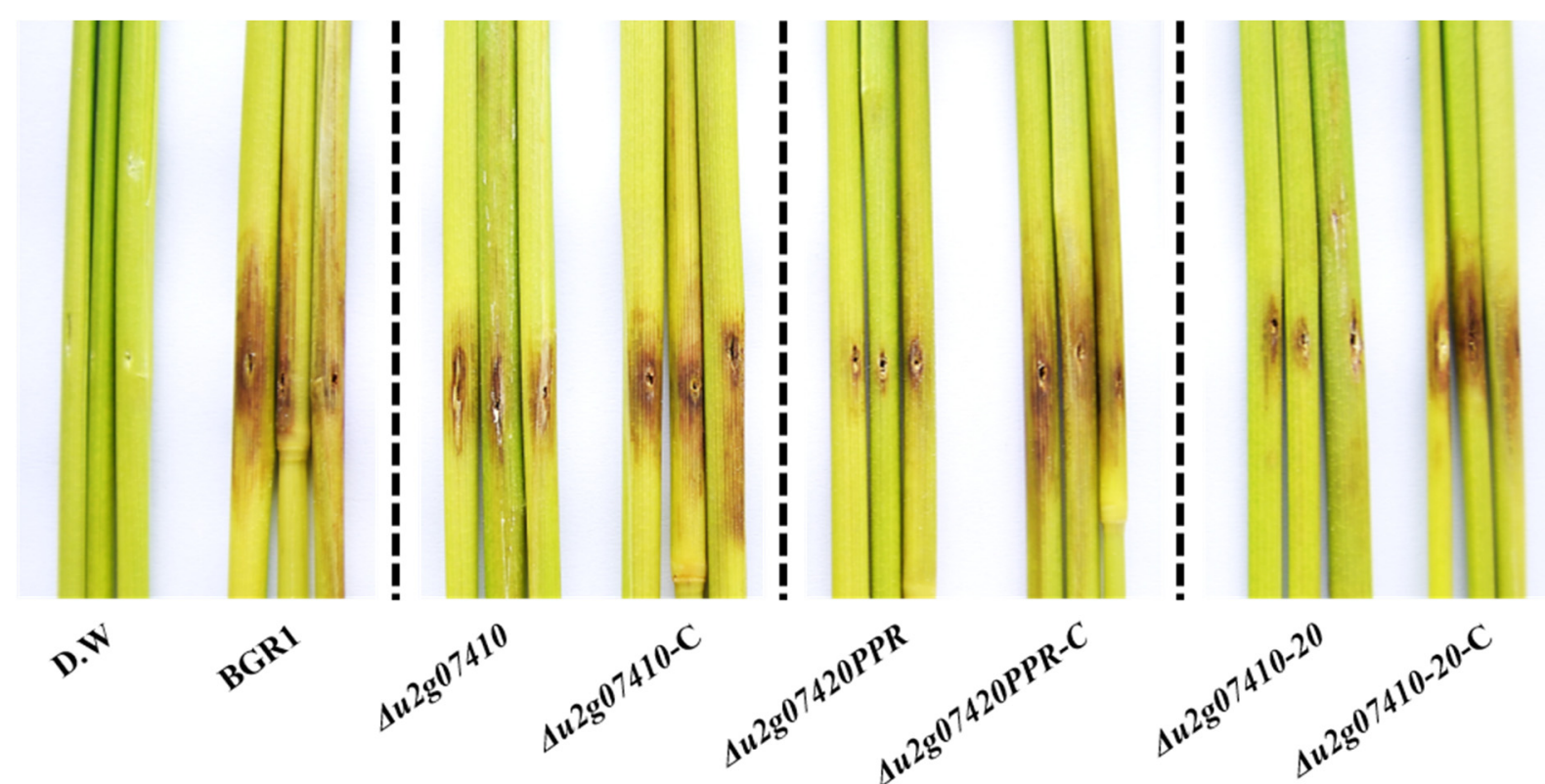

Figure 5. In vivo pathogenicity assay at the vegetative stage to assess the virulence of $\Delta u 2 g 07410$, $\triangle u 2 g 07420 P P R$, and $\Delta u 2 g 07410-20$. Wild-type and mutant strains were inoculated in the stems of rice plants. Representative of three replicates. Disease symptoms at 8 days post-inoculation. Distilled water (D.W) was used as the negative control.

\section{Discussion}

T6SS has evolved into the most complex secretory pathway because of its key role during interactions with the surroundings [29]. T6SS is known to have two distinct functional features: a prokaryotic targeting system with antibacterial effects manifesting as bacterial interactions, and a eukaryotic targeting system demonstrating bacterial virulence as eukaryotic interactions. T6SS is a proteinaceous machine with 13 core components. The core genes of T6SS are organized into genomic clusters. In addition to the core components, the gene cluster of T6SS additionally encodes accessory genes functionally associated with T6SS [20,30-34]. These accessory proteins are either involved in the secretion of T6SSdependent effectors or regulate the assembly and activity of T6SS [35]. The functions of most T6SS accessory proteins have not been identified. In particular, the protein comprising the DUF2169 domain exists as a single-domain protein with only the DUF2169 domain; however, some are multi-domain proteins with additional domains as small portions at the C-terminus [36]. The most common additional domain of the DUF2169 domain-containing protein is the pentapeptide repeats region, which has unknown functions [36].

The DUF2169 domain-containing protein of a single-domain protein can be involved in the functional role of T6SS as an adapter, even if there is no additional domain at the C-terminus. For example, a single DUF2169 domain-containing protein encoded by atu3641 in Agrobacterium tumefaciens C58 is a T6SS adapter associated with Tde2, an effector of T6SS, and contributes to the secretion of Tde2 [22].

B. glumae BGR1 has a DUF2169 domain-containing protein encoded by two genes, bglu_1g23320 and $b g l u \_2 g 07420$, on its genome. These genes are located downstream of the $\operatorname{vgr} G$. The genes which encode T6SS accessory proteins, such as adaptors, are often found downstream of $\operatorname{vgrG}$ [27]. bglu_1g23320 encodes a single-domain protein with only the DUF2169 domain. The bglu_1g23320 gene was not included in any T6SS gene cluster of $B$. glumae BGR1 (Figure 1). Some T6SS accessory genes and its upstream gene, $\operatorname{vgr} G$, are located outside the T6SS gene cluster and borrow the core components of T6SS [37]. However, $\Delta u 1 g 23320$ did not show attenuated virulence in rice plants (Figure 3). T6SS adapters, such as the DUF2169 domain-containing protein, enable secretion by loading specific effectors into cognate $\operatorname{VgrG}$ (Valine glycine repeat $\mathrm{G}$ ) without affecting the function of T6SS [22,27,35]. Therefore, the DUF2169 domain-containing protein encoded by bglu_1g23320, which is not 
included in any T6SS gene cluster of B. glumae BGR1, is not involved in a specific effector that directly causes pathogenicity in rice plants, or may remain as a non-functional T6SS accessory protein.

In contrast to $b g l u \_1 g 23320, b g l u \_2 g 07420$ encodes a multi-domain protein with the DUF2169 domain and pentapeptide repeats region as an additional domain at the Cterminus. Additionally, bglu_2g07420 is included in the T6SS group_5 gene cluster of B. glumae BGR1. bglu_2g07420 encodes a DUF2169 domain-containing protein and is present in the gene cluster of T6SS group_5; therefore, a DUF2169 domain-containing protein encoded by bglu_2g07420 can be considered as a T6SS accessory protein of T6SS group_5 in B. glumae BGR1. The T6SS group_5 of B. glumae BGR1 is involved in bacterial virulence in rice plants [3]. In addition, the T6SS-5 gene cluster of $B$. pseudomallei and $B$. thailandensis belonging to the eukaryotic system has $\operatorname{tag} A / B-5$, which encodes the DUF2169 domain-containing protein [38]. Additionally, TagA/B-5 of B. thailandensis is a multidomain protein composed of the DUF2169 domain and pentapeptide repeats region as an additional domain at the C-terminus, which is essential for full virulence in multinucleated giant cells [39]. Thus, the DUF2169 domain-containing protein encoded by bglu_2g07420 was also hypothesized to be essential for bacterial virulence as a T6SS accessory protein of the T6SS group_5 in B. glumae BGR1.

The single-domain protein containing DUF2169 can function by itself; therefore, $\Delta u 2 g 07420 D U F 2169$ was generated by deleting only DUF2169 of the DUF2169 domaincontaining protein without causing any problem in the frameshift of $b g l u \_2 g 07420$. Furthermore, an in vivo pathogenicity assay was performed with this strain. The attenuated virulence of $\Delta u 2 g 07420 D U F 2169$ indicated that the DUF2169 domain of the DUF2169 domain-containing protein encoded by $b g l u \_2 g 07420$ is required for bacterial virulence in rice plants. $\triangle u 2 g 07420 P P R$ was generated to determine whether the DUF2169 domaincontaining protein encoded by $b g l u \_2 g 07420$ can fully function as a T6SS accessory protein even in the absence of an additional domain. An in vivo pathogenicity assay was also performed using this strain. Similarly to $\Delta u 2 g 07420 D U F 2169$, the attenuated virulence of $\triangle u 2 g 07420 P P R$ indicated that the DUF2169 domain-containing protein encoded by bglu_2g07420 requires not only the DUF2169 domain, but also the additional C-terminal domain, the pentapeptide repeats region, to enable bacterial virulence in rice plants. Therefore, the DUF2169 domain-containing protein encoded by bglu_2g07420 is a functional T6SS accessory protein consisting of multiple domains having the DUF2169 domain and an additional C-terminal pentapeptide repeats region.

bglu_2g07410, located downstream of $b g l u \_2 g 07420$ which encodes the additional C-terminal domain of the pentapeptide repeats region, encodes only the pentapeptide repeats region. The protein containing only the pentapeptide repeats region might also contribute to bacterial virulence in rice plants with the DUF2169 domain-containing protein having an additional C-terminal pentapeptide repeats region. Indeed, $\Delta u 2 g 07410$ also showed attenuated virulence in rice plants. The attenuated virulence of $\Delta u 2 g 07410$ indicates that the pentapeptide repeats protein, encoded by $b g l u \_2 g 07410$, also contributes to bacterial virulence in rice plants. Pentapeptide repeats proteins are widely distributed in both prokaryotes and eukaryotes [40]. These proteins may be responsible for targeting, or for structural functions, rather than enzymatic activity [40]. These proteins have a superhelical structure and can participate in protein-protein interactions without conferring biological functions [40]. For example, the pentapeptide repeats regions of MfpA and Qnr have a superhelical structure, mimicking the B-form of DNA and inhibiting DNA gyrase, conferring resistance to fluoroquinolone [41-44]. However, the functions or roles of pentapeptide repeats proteins are not fully understood.

Interestingly, the type III secretion system-dependent effector, PipB2, in Salmonella spp. contains the pentapeptide repeats region, which directly interacts with kinesin- 1 and contributes to the formation of Salmonella-induced filaments. However, the exact function and role of the pentapeptide repeats region in PipB2 is also unclear [41-43]. Interestingly, in the results of the in vivo pathogenicity assay at the reproductive stage, the severely 
attenuated virulence by $\Delta u 2 g 07410-20$ was shown to be a synergistic effect of the two T6SS accessory proteins encoded by bglu_2g07410 and bglu_2g07420. B. glumae BGR1 mainly causes BPB as a serious symptom in the reproductive stage of rice plants, but can also cause diseases at any developmental stage, such as seed rot, stunting in seedlings, and sheath rot in the vegetative stage. Neopseudocercosporella capsellae, a plant pathogen, causes disease in canola, and the incidence and the severity of the disease are determined by the growth stage of the host plant in which this pathogen invasion occurs [45]. Therefore, the results of the in vivo pathogenicity assay at the reproductive stage show that B. glumae BGR1 induces $\mathrm{BPB}$, with distinct functions of these two T6SS accessory proteins involved in bacterial virulence at the reproductive stage.

In the current study, we obtained genes encoding the DUF2169 domain from the genome of B. glumae BGR1 and investigated whether the genes encoding the DUF2169 domain-containing proteins consisting of a single domain or multi-domain affect bacterial virulence to rice plants. Of the two genes encoding the DUF2169 domain-containing protein, bglu_1g23320 and bglu_2g07420, only the latter was involved in bacterial virulence. We discovered two genes encoding the T6SS accessory proteins in the gene cluster of T6SS group_5 in B. glumae BGR1: bglu_2g07420 and bglu_2g07410. Our results show that the DUF2169 domain-containing protein encoded in bglu_2g074720 is a multi-domain protein that requires both the DUF2169 domain and the pentapeptide repeats region to contribute to bacterial virulence. T6SS accessory protein, having a DUF2169 domain, is a well-known T6SS adaptor. The DUF2169 domain-containing protein encoded by bglu_2g07420 could be considered as a potential T6SS adaptor; however, additional experimental evidence, such as interactions with VgrG or specific T6SS-dependent effectors, is required to confirm its function as a T6SS adaptor. Furthermore, we showed that another T6SS accessory protein, encoded by bglu_2g07410 located downstream of bglu_2g07420, is involved in bacterial virulence as the pentapeptide repeats protein. To the best of our knowledge, this is the first report on the identification of the T6SS accessory proteins, the DUF2169 domain-containing protein, and the pentapeptide repeats protein, belonging to T6SS group_ 5 of B. glumae BGR1 and involved in bacterial virulence. Novel T6SS accessory proteins involved in the bacterial virulence of B. glumae BGR1 can be usefully employed to understand the functional activity of T6SS.

\section{Materials and Methods}

\subsection{Bacterial Strains, Plasmids, and Growth Conditions}

All bacterial strains and plasmids used in this study are detailed in Table 2. The wild-type BGR1, mutant strains, and E. coli were cultured on Luria Bertani (LB) agar and in broth medium at $37^{\circ} \mathrm{C}$ with shaking at $200 \mathrm{rpm}$. The growth curve of bacterial strains was determined every $2 \mathrm{~h}$ by measuring the optical density at $600 \mathrm{~nm}\left(\mathrm{OD}_{600}\right)$ using a UV-1800 spectrophotometer (Shimadzu, Kyoto, Japan). When required, LB media were supplemented with antibiotics at concentrations of $100 \mu \mathrm{g} / \mathrm{mL}$ (rifampicin) and $50 \mu \mathrm{g} / \mathrm{mL}$ (kanamycin).

Table 2. Bacterial strains and plasmids used in this study.

\begin{tabular}{|c|c|c|}
\hline Name & Characteristics & Source \\
\hline \multicolumn{3}{|c|}{ Bacterial strains } \\
\hline \multicolumn{3}{|l|}{ B. glumae } \\
\hline BGR1 & Burkholderia glumae isolate from rice, wild-type, $\operatorname{Rif}^{\mathrm{r}}$ * & [4] \\
\hline$\Delta u 2 g 07410$ & BGR1 derivative, deletion of 824 bp within bglu_2g07410 & This study \\
\hline$\Delta u 2 g 07420 \_D U F 2169$ & $\begin{array}{l}\text { BGR1 derivative, deletion of } 1466 \text { bp of the DUF2169 domain region within } \\
\qquad \text { bglu_2g07420 }\end{array}$ & This study \\
\hline$\triangle u 2 g 07420 \_P P R$ & $\begin{array}{l}\text { BGR1 derivative, deletion of } 927 \text { bp of the pentapeptide repeats region within } \\
\qquad \text { bglu_2g0720 }\end{array}$ & This study \\
\hline
\end{tabular}


Table 2. Cont.

\begin{tabular}{|c|c|c|}
\hline Name & Characteristics & Source \\
\hline$\Delta u 2 g 07410-20$ & $\begin{array}{c}b g l u \_2 g 07410 \text { and pentapeptide repeats region of } b g l u \_2 g 07420 \text { double deletion } \\
\text { mutant derived from BGR1 }\end{array}$ & This study \\
\hline$\Delta u 1 g 23320$ & BGR1 derivative, deletion of 951 bp within bglu_1g23320 & This study \\
\hline$\Delta u 2 g 07410-\mathrm{C}$ & BGR1 bglu_2g07410 deletion mutant containing pBu2g07410 & This study \\
\hline$\Delta u 2 g 07420 \_D U F 2169-C$ & $\begin{array}{l}\text { BGR1 bglu_2g07420 deletion mutant }\left(\Delta u 2 g 07420 \_D U F 2169\right) \text { containing } \\
\text { pBu2g07420 }\end{array}$ & This study \\
\hline$\triangle u 2 g 07420 \_P P R-C$ & BGR1 bglu_2g07420 deletion mutant $\left(\triangle u 2 g 07420 \_P P R\right)$ containing $\mathrm{pB} u 2 g 07420$ & This study \\
\hline$\Delta u 2 g 07410-20-\mathrm{C}$ & $\begin{array}{l}\text { BGR1 bglu_2g07410-20 double deletion mutant }(\Delta u 2 g 07410-20) \text { containing } \\
\text { pBu2g07410 and } \mathrm{pBu} 2 g 07420\end{array}$ & This study \\
\hline \multicolumn{3}{|c|}{ 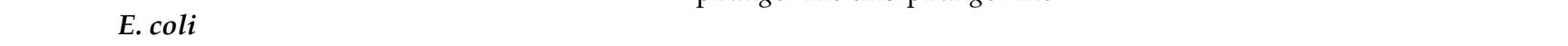 } \\
\hline E. coli $\mathrm{DH} 5 \alpha \lambda$ pir & $\begin{array}{c}\mathrm{F}^{-} \text {80dlacZDM15 (lacZYA-argF) U169 recA1 endA1hsdR17 (rk-, mk+) phoAsupE44 } \\
\text {-thi-1 gyrA96 relA1 }\end{array}$ & Lab collection \\
\hline \multicolumn{3}{|l|}{ Plasmids } \\
\hline $\mathrm{pK} 18 m o b s a c B$ & Allelic exchange suicide vector, $s a c B \mathrm{Km}^{\mathrm{r}} *$ & [47] \\
\hline pK18u2g07410 & $\begin{array}{l}\text { For constructing bglu_2g07410 KO mutant, pK18mobsacB:: LR fragment of } \\
\text { bglu_2g07410 region restricted by EcoRI-HindIII }\end{array}$ & This study \\
\hline pK18u2g07420DUF2169 & $\begin{array}{l}\text { For constructing the DUF2169 domain in the bglu_2g07420 KO mutant, } \\
\text { pK18mobsacB:: LR fragment of the DUF2169 domain region in bglu_2g07420 } \\
\text { restricted by EcoRI-HindIII }\end{array}$ & This study \\
\hline pK18u2g07420PPR & $\begin{array}{l}\text { For constructing pentapeptide repeats region in the } b g l u \_2 g 07420 \mathrm{KO} \text { mutant, } \\
\text { pK18mobsacB::LR fragment of pentapeptide repeats region in bglu_2g07420 } \\
\text { restricted by BamHI-HindIII }\end{array}$ & This study \\
\hline pK18u1g23320 & $\begin{array}{l}\text { For constructing bglu_1g23320 KO mutant, pK18mobsacB:: LR fragment of } \\
\text { bglu_1g23320 region restricted by BamHI-HindIII }\end{array}$ & This study \\
\hline pBBR1MCS2 & Broad-host-range plasmid, $\mathrm{Km}^{\mathrm{r} *}$, used to construct complementation strains. & [48] \\
\hline $\mathrm{pB} u 2 g 07410$ & $\begin{array}{l}\text { For constructing the } \Delta u 2 g 07410 \text { complementation strain, pBBR1MCS2::CDS of } \\
\qquad \text { bglu_2g07410 }\end{array}$ & This study \\
\hline $\mathrm{pB} u 2 g 07420$ & $\begin{array}{l}\text { For constructing the } \Delta u 2 g 07420 \_D U F 2169 \text { and } \Delta u 2 g 07420 \_P P R \\
\text { complementation strain, pBBR1MCS2::CDS of } b g l u \_2 g 07420\end{array}$ & This study \\
\hline
\end{tabular}

${ }^{*}$ Abbreviations: Rif ${ }^{\mathrm{r}}$-rifampicin resistance; $\mathrm{Km}^{\mathrm{r}}$ — kanamycin resistance.

4.2. Construction of Markerless Deletion Mutants and Their Complemetation Strains in B. glumae BGR1

All primer pairs used for the construction of deletion mutants are shown in Supplementary Table S3. Plasmids, including pK18u1g23320, pK18u2g07410, pK18u2g07420DUF2169, and pk18u2g07420PPR, harboring portions of the target gene upstream (L fragment) and downstream ( $R$ fragment), were prepared. The $L$ and $R$ fragments were designed according to the Gibson assembly reaction. The $\mathrm{L}$ and $\mathrm{R}$ fragments were ligated by secondary PCR using LF and RR primer pairs. To construct pK18u1g23320, the ligated LR fragments and pK18mobsacB were digested with BamHI-HindIII (NEB, Ipswich, MA, USA). To construct pK18u2g07410, the ligated LR fragments and pK18mobsacB were digested with EcoRI and HindIII. To construct pK18u2g07420DUF2169, the ligated LR fragments and pK18mobsacB were digested with EcoRI and HindIII. To construct pK18u2g07420PPR, the ligated LR fragments and pK18mobsacB were digested with BamHI and HindIII. Competent E. coli DH5a cells were transformed with recombinant plasmids and selected in kanamycin-containing media. Recombinant plasmids were transformed into E. coli S17-1 cells. Recombinant plasmids were transformed into B. glumae BGR1 by conjugation through co-culture with E. coli S17-1 on LB agar plates. The first crossover was selected on media containing kanamycin $(60 \mu \mathrm{g} / \mathrm{mL})$ and rifampicin $(100 \mu \mathrm{g} / \mathrm{mL})$. The first crossover of colonies was confirmed by PCR using the primer pairs of genes,_UP_F and pK18_DOWN_R. The selected cells underwent a second crossover in LB broth containing kanamycin $(60 \mu \mathrm{g} / \mathrm{mL})$. The second crossover in the cells was selected on LB agar, containing 30\% sucrose and rifampicin $(100 \mu \mathrm{g} / \mathrm{mL})$. Finally, single deletions of bglu_1g23320, bglu_2g07410, the DUF2169 domain region, and the pentapeptide repeats region of bglu_2g07420 gene were confirmed 
by PCR using the primer sets for gene_UP_F and gene_DOWN_R. The double deletion mutant, $\Delta 2 g 07410-20$, was constructed based on single $\Delta 2 g 07410$ deletion mutants using the above-mentioned methods.

For complementation, the entire open reading frame of $b g l u \_2 g 07410$ and $b g l u \_2 g 07420$ genes was amplified by PCR using the primer pairs Cgene_F and Cgene_R. The amplified DNA fragments were cloned into the broad-host-range expression vector, pBBR1MCS2. The cloned vectors, $\mathrm{pB} u 2 g 07410$ and/or $\mathrm{pB} u 2 g 07420$, were conjugated into single and double deletion mutant strains by co-culturing with S17-1. Finally, complementation strains of single and double deletion mutants were selected on media containing rifampicin $(100 \mu \mathrm{g} / \mathrm{mL})$ and kanamycin $(60 \mu \mathrm{g} / \mathrm{mL})$ and were confirmed by PCR using primer pairs of pB_UP_F and pB_DOWN_R.

\subsection{In Vivo Pathogenicity Assay at the Vegetative and Reproductive Stages of Rice Plants}

To examine the bacterial virulence of putative T6SS accessory proteins in B. glumae BGR1 at the vegetative and reproductive stages of rice plants, cultured bacterial cells in the mid-logarithmic phase were harvested by centrifugation, washed, and resuspended in distilled water. Thereafter, the optical density of the bacterial suspensions was adjusted to $\mathrm{OD}_{600 \mathrm{~nm}}=0.8$. The rice plants (Oryza sativa L. Saeilmi) used in this experiment were grown under greenhouse conditions (average $30^{\circ} \mathrm{C}$ in the day, and $25^{\circ} \mathrm{C}$ at night). Subsequently, the stems of vegetative-stage rice plants were inoculated with bacterial suspensions using a syringe and grown for 8 days. Disease severity was observed in the inoculated area. Suspensions for each bacterial strain were prepared and adjusted to $\mathrm{OD}_{600 \mathrm{~nm}}=0.5$ to confirm bacterial panicle blight disease at the rice reproductive stage. At the flowering stage, the rice panicles were inoculated via dipping into $50 \mathrm{~mL}$ bacterial suspensions for $1 \mathrm{~min}$. At $8 \mathrm{dpi}$, the disease severity of the rice panicles in individual rice plants was evaluated using the following scale: 0 -healthy panicle; $1-0-20 \%$ diseased panicle; $2-20-40 \%$ diseased panicle; $3-40-60 \%$ diseased panicle; $4-40-80 \%$ diseased panicle; $5-80-100 \%$ diseased panicle. Disease severity was calculated using the following formula: disease severity $=\Sigma$ (number of samples per rating $\times$ rating value)/total number of panicles.

\subsection{Statistical Analysis}

All experiments were conducted at least three times, with at least three replicates. Analyses of the disease severity scores were conducted with at least three replicates of individual rice plants. The mean disease severity scores between two groups were compared using the least significant difference test according to Tukey's HSD assay.

Supplementary Materials: The following supporting information can be downloaded at: https:/ / www.mdpi.com/article/10.3390/plants11010034/s1, Table S1: Analysis of deletion site in $\Delta u 1 g 23320$, Table S2: Analysis of deletion site in $\Delta u 2 g 07410$,Table S3: Analysis of deletion site in $\Delta u 2 g 07420 D U F 2169$ and $\triangle u 2 g 07420 P P R$, Table S4: Oligonucleotide primers used in this study.

Author Contributions: Conceptualization, N.K. and Y.-S.S.; methodology, N.K., G.H. and J.P.; formal analysis, H.J. and H.-H.L.; data curation, N.K. and H.J.; writing-original draft preparation, N.K.; writing-review and editing, G.H. and Y.-S.S.; project administration, Y.-S.S.; funding acquisition, N.K. and Y.-S.S. All authors have read and agreed to the published version of the manuscript.

Funding: This study was supported by the BK21 Four Program of Pusan National University (N.K.), and a two-year Research Grant from Pusan National University (Y.-S.S.).

Institutional Review Board Statement: Not applicable.

Informed Consent Statement: Not applicable.

Data Availability Statement: Data sharing is not applicable to this article.

Acknowledgments: We appreciate the technical support provided Do-Young Lee.

Conflicts of Interest: The authors declare no conflict of interest. 


\section{References}

1. Goto, K. New bacterial diseases of rice-bacterial brown stripe and bacterial grain rot. Ann. Phytopathol. Soc. Jpn. 1956, $21,46-47$.

2. Goto, T.; Nishiyama, K.; Ohata, K.-I. Bacteria causing grain rot of rice. Jpn. J. Phytopathol. 1987, 53, 141-149. [CrossRef]

3. Kim, N.; Kim, J.J.; Kim, I.; Mannaa, M.; Park, J.; Kim, J.; Lee, H.; Lee, S.; Park, D.; Sul, W.J.; et al. Type VI secretion systems of plant-pathogenic Burkholderia glumae BGR1 play a functionally distinct role in interspecies interactions and virulence. Mol. Plant Pathol. 2020, 21, 1055-1069. [CrossRef] [PubMed]

4. Jeong, Y.; Kim, J.; Kim, S.; Kang, Y.; Nagamatsu, T.; Hwang, I. Toxoflavin Produced by Burkholderia glumae Causing Rice Grain Rot Is Responsible for Inducing Bacterial Wilt in Many Field Crops. Plant Dis. 2003, 87, 890-895. [CrossRef]

5. $\quad$ Iiyama, K.; Furuya, N.; Takanami, Y.; Matsuyama, N. A Role of Phytotoxin in Virulence of Pseudomonas glumae Kurita et Tabei. Jpn. J. Phytopathol. 1995, 61, 470-476. [CrossRef]

6. Suzuki, F.; Sawada, H.; Azegami, K.; Tsuchiya, K. Molecular characterization of the tox operon involved in toxoflavin biosynthesis of Burkholderia glumae. J. Gen. Plant Pathol. 2004, 70, 97-107. [CrossRef]

7. Kim, J.; Kang, Y.; Choi, O.; Jeong, Y.; Jeong, J.-E.; Lim, J.Y.; Kim, M.; Moon, J.S.; Suga, H.; Hwang, I. Regulation of polar flagellum genes is mediated by quorum sensing and FlhDC in Burkholderia glumae. Mol. Microbiol. 2007, 64, 165-179. [CrossRef]

8. Kang, Y.; Kim, J.; Kim, S.; Kim, H.; Lim, J.Y.; Kim, M.; Kwak, J.; Moon, J.S.; Hwang, I. Proteomic analysis of the proteins regulated by HrpB from the plant pathogenic bacterium Burkholderia glumae. Proteomics 2008, 8, 106-121. [CrossRef]

9. Lee, J.; Park, J.; Kim, S.; Park, I.; Seo, Y.-S. Differential regulation of toxoflavin production and its role in the enhanced virulence of Burkholderia gladioli. Mol. Plant Pathol. 2016, 17, 65-76. [CrossRef]

10. Jung, B.; Park, J.; Kim, N.; Li, T.; Kim, S.; Bartley, L.E.; Kim, J.; Kim, I.; Kang, Y.; Yun, K.; et al. Cooperative interactions between seed-borne bacterial and air-borne fungal pathogens on rice. Nat. Commun. 2018, 9, 31. [CrossRef]

11. Mougous, J.D.; Cuff, M.E.; Raunser, S.; Shen, A.; Zhou, M.; Gifford, C.A.; Goodman, A.L.; Joachimiak, G.; Ordoñez, C.L.; Lory, S.; et al. A Virulence Locus of Pseudomonas aeruginosa Encodes a Protein Secretion Apparatus. Science 2006, 312, 1526-1530. [CrossRef] [PubMed]

12. Pukatzki, S.; Ma, A.; Sturtevant, D.; Krastins, B.; Sarracino, D.; Nelson, W.; Heidelberg, J.; Mekalanos, J.J. Identification of a conserved bacterial protein secretion system in Vibrio cholerae using the Dictyostelium host model system. Proc. Natl. Acad. Sci. USA 2006, 103, 1528-1533. [CrossRef]

13. Boyer, F.; Fichant, G.; Berthod, J.; Vandenbrouck, Y.; Attree, I. Dissecting the bacterial type VI secretion system by a genome wide in silico analysis: What can be learned from available microbial genomic resources? BMC Genom. 2009, 10, 104. [CrossRef]

14. Ma, L.-S.; Lin, J.-S.; Lai, E.-M. An IcmF Family Protein, ImpL M, Is an Integral Inner Membrane Protein Interacting with ImpK L, and Its Walker A Motif Is Required for Type VI Secretion System-Mediated Hcp Secretion in Agrobacterium tumefaciens. J. Bacteriol. 2009, 191, 4316-4329. [CrossRef] [PubMed]

15. Filloux, A.; Freemont, P. Structural biology: Baseplates in contractile machines. Nat. Microbiol. 2016, 1, 16104. [CrossRef] [PubMed]

16. Nazarov, S.; Schneider, J.P.; Brackmann, M.; Goldie, K.N.; Stahlberg, H.; Basler, M. Cryo- EM reconstruction of Type VI secretion system baseplate and sheath distal end. EMBO J. 2017, 37, e97103. [CrossRef]

17. Pukatzki, S.; Ma, A.T.; Revel, A.T.; Sturtevant, D.; Mekalanos, J.J. Type VI secretion system translocates a phage tail spike-like protein into target cells where it cross-links actin. Proc. Natl. Acad. Sci. USA 2007, 104, 15508-15513. [CrossRef]

18. Pell, L.G.; Kanelis, V.; Donaldson, L.; Howell, P.L.; Davidson, A.R. The phage major tail protein structure reveals a common evolution for long-tailed phages and the type VI bacterial secretion system. Proc. Natl. Acad. Sci. USA 2009, 106, $4160-4165$. [CrossRef]

19. Silverman, J.M.; Brunet, Y.R.; Cascales, E.; Mougous, J.D. Structure and Regulation of the Type VI Secretion System. Annu. Rev. Microbiol. 2012, 66, 453-472. [CrossRef]

20. Shneider, M.M.; Buth, S.; Ho, B.; Basler, M.; Mekalanos, J.J.; Leiman, P.G. PAAR-repeat proteins sharpen and diversify the type VI secretion system spike. Nature 2013, 500, 350-353. [CrossRef]

21. Brackmann, M.; Nazarov, S.; Wang, J.; Basler, M. Using Force to Punch Holes: Mechanics of Contractile Nanomachines. Trends Cell Biol. 2017, 27, 623-632. [CrossRef]

22. Bondage, D.D.; Lin, J.-S.; Ma, L.-S.; Kuo, C.-H.; Lai, E.-M. VgrG C terminus confers the type VI effector transport specificity and is required for binding with PAAR and adaptor-effector complex. Proc. Natl. Acad. Sci. USA 2016, 113, E3931-E3940. [CrossRef] [PubMed]

23. Cianfanelli, F.R.; Alcoforado Diniz, J.; Guo, M.; De Cesare, V.; Trost, M.; Coulthurst, S.J. VgrG and PAAR Proteins Define Distinct Versions of a Functional Type VI Secretion System. PLoS Pathog. 2016, 12, e1005735. [CrossRef] [PubMed]

24. Flaugnatti, N.; Le, T.T.H.; Canaan, S.; Aschtgen, M.-S.; Nguyen, V.S.; Blangy, S.; Kellenberger, C.; Roussel, A.; Cambillau, C.; Cascales, E.; et al. A phospholipase A1 antibacterial Type VI secretion effector interacts directly with the C-terminal domain of the VgrG spike protein for delivery. Mol. Microbiol. 2016, 99, 1099-1118. [CrossRef] [PubMed]

25. Lien, Y.-W.; Lai, E.-M. Type VI Secretion Effectors: Methodologies and Biology. Front. Cell. Infect. Microbiol. 2017, 7, 254. [CrossRef] [PubMed]

26. Unterweger, D.; Kostiuk, B.; Pukatzki, S. Adaptor Proteins of Type VI Secretion System Effectors. Trends Microbiol. 2017, 25, 8-10. [CrossRef] 
27. Unterweger, D.; Kostiuk, B.; Ötjengerdes, R.; Wilton, A.; Diaz-Satizabal, L.; Pukatzki, S. Chimeric adaptor proteins translocate diverse type VI secretion system effectors in Vibrio cholerae. EMBO J. 2015, 34, 2198-2210. [CrossRef]

28. Liang, X.; Moore, R.; Wilton, M.; Wong, M.J.Q.; Lam, L.; Dong, T.G. Identification of divergent type VI secretion effectors using a conserved chaperone domain. Proc. Natl. Acad. Sci. USA 2015, 112, 9106-9111. [CrossRef]

29. Nguyen, T.T.; Lee, H.-H.; Park, I.; Seo, Y.-S. Genome-Wide Analysis of Type VI System Clusters and Effectors in Burkholderia Species. Plant Pathol. J. 2018, 34, 11-22. [CrossRef]

30. Leiman, P.; Basler, M.; Ramagopal, U.A.; Bonanno, J.B.; Sauder, J.M.; Pukatzki, S.; Burley, S.; Almo, S.C.; Mekalanos, J.J. Type VI secretion apparatus and phage tail-associated protein complexes share a common evolutionary origin. Proc. Natl. Acad. Sci. USA 2009, 106, 4154-4159. [CrossRef]

31. Felisberto-Rodrigues, C.; Durand, E.; Aschtgen, M.-S.; Blangy, S.; Ortiz-Lombardía, M.; Douzi, B.; Cambillau, C.; Cascales, E. Towards a Structural Comprehension of Bacterial Type VI Secretion Systems: Characterization of the TssJ-TssM Complex of an Escherichia coli Pathovar. PLoS Pathog. 2011, 7, e1002386. [CrossRef] [PubMed]

32. Kapitein, N.; Bönemann, G.; Pietrosiuk, A.; Seyffer, F.; Hausser, I.; Locker, J.K.; Mogk, A. ClpV recycles VipA/VipB tubules and prevents non-productive tubule formation to ensure efficient type VI protein secretion. Mol. Microbiol. 2013, 87, 1013-1028. [CrossRef] [PubMed]

33. Silverman, J.M.; Agnello, D.M.; Zheng, H.; Andrews, B.T.; Li, M.; Catalano, C.E.; Gonen, T.; Mougous, J.D. Haemolysin Coregulated Protein Is an Exported Receptor and Chaperone of Type VI Secretion Substrates. Mol. Cell 2013, 51, 584-593. [CrossRef] [PubMed]

34. Taylor, N.M.I.; Prokhorov, N.; Guerrero-Ferreira, R.; Shneider, M.M.; Browning, C.; Goldie, K.N.; Stahlberg, H.; Leiman, P. Structure of the T4 baseplate and its function in triggering sheath contraction. Nature 2016, 533, 346-352. [CrossRef]

35. Diniz, J.A.; Coulthurst, S.J. Intraspecies Competition in Serratia marcescens Is Mediated by Type VI-Secreted Rhs Effectors and a Conserved Effector-Associated Accessory Protein. J. Bacteriol. 2015, 197, 2350-2360. [CrossRef]

36. Liu, Y.; Zhang, Z.; Wang, F.; Li, D.-D.; Li, Y.-Z. Identification of type VI secretion system toxic effectors using adaptors as markers Comput. Struct. Biotechnol. J. 2020, 18, 3723-3733. [CrossRef] [PubMed]

37. Zong, B.; Zhang, Y.; Wang, X.; Liu, M.; Zhang, T.; Zhu, Y.; Zheng, Y.; Hu, L.; Li, P.; Chen, H.; et al. Characterization of multiple type-VI secretion system (T6SS) VgrG proteins in the pathogenicity and antibacterial activity of porcine extra-intestinal pathogenic Escherichia coli. Virulence 2019, 10, 118-132. [CrossRef]

38. Shalom, G.; Shaw, J.G.; Thomas, M.S. In vivo expression technology identifies a type VI secretion system locus in Burkholderia pseudomallei that is induced upon invasion of macrophages. Microbiology 2007, 153, 2689-2699. [CrossRef]

39. Hopf, V.; Göhler, A.; Eske-Pogodda, K.; Bast, A.; Steinmetz, I.; Breitbach, K. BPSS1504, a Cluster 1 Type VI Secretion Gene, Is Involved in Intracellular Survival and Virulence of Burkholderia pseudomallei. Infect. Immun. 2014, 82, 2006-2015. [CrossRef]

40. Bateman, A.; Murzin, A.G.; Teichmann, S.A. Structure and distribution of pentapeptide repeats in bacteria. Protein Sci. 1998, 7, 1477-1480. [CrossRef]

41. Tran, J.H.; Jacoby, G.A.; Hooper, D.C. Interaction of the Plasmid-Encoded Quinolone Resistance Protein QnrA with Escherichia coli Topoisomerase IV. Antimicrob. Agents Chemother. 2005, 49, 3050-3052. [CrossRef] [PubMed]

42. Henry, T.; Couillault, C.; Rockenfeller, P.; Boucrot, E.; Dumont, A.; Schroeder, N.; Hermant, A.; Knodler, L.; Lécine, P.; SteeleMortimer, O.; et al. The Salmonella effector protein PipB2 is a linker for kinesin-1. Proc. Natl. Acad. Sci. USA 2006, 103, 13497-13502. [CrossRef] [PubMed]

43. Vetting, M.W.; Hegde, S.S.; Fajardo, J.E.; Fiser, A.; Roderick, S.L.; Takiff, H.E.; Blanchard, J.S. Pentapeptide Repeat Proteins. Biochemistry 2003, 45, 1-10. [CrossRef]

44. Zhang, R.; Kennedy, M. Current Understanding of the Structure and Function of Pentapeptide Repeat Proteins. Biomolecules 2021, 11, 638. [CrossRef] [PubMed]

45. Murtza, T.; You, M.P.; Barbetti, M.J. Canola Growth Stage at Time of Infection Determines Magnitude of White Leaf Spot (Neopseudocercosporella capsellae) Impact. Plant Dis. 2021, 105, 1515-1521. [CrossRef] [PubMed]

46. Kalogeraki, V.S.; Winans, S.C. Suicide plasmids containing promoterless reporter genes can simultaneously disrupt and create fusions to target genes of diverse bacteria. Gene 1997, 188, 69-75. [CrossRef]

47. Schäfer, A.; Tauch, A.; Jsger, W.; Kalinowski, J.; Thierbachb, G.; Piihler, A. Small mobilizable multi-purpose cloning vectors derived from the Escherichia coli plasmids pK18 and pK19: Selection of defined deletions in the chromosome of Corynebacterium glutamicum. Gene 1994, 145, 49-5201. [CrossRef]

48. Kovach, M.E.; Elzer, P.H.; Hill, D.S.; Robertson, G.T.; Farris, M.A.; Roop, R.M., II; Peterson, K.M. Four new derivatives of the broad-host-range cloning vector pBBR1MCS, carrying different antibiotic-resistance cassettes. Gene 1995, 166, 175-176. [CrossRef] 